(C) 2016 IEEE. Personal use of this material is permitted. Permission from IEEE must be obtained for all other uses, in any current or future media, including reprinting/republishing this material for advertising or promotional purposes, creating new collective works, for resale or redistribution to servers or lists, or reuse of any copyrighted component of this work in other works. 


\section{Setting the Maximum Import Net Transfer Capacity under Extreme RES Integration Scenarios}

\author{
M.A. Matos, RJ. Bessa, C. Gonçalves, L. Cavalcante. \\ V. Miranda \\ INESC TEC and Faculty of Engineering, University of \\ Porto, Portugal \\ mam@fe.up.pt, cssg@inesctec.pt, \\ ricardo.j.bessa@inesctec.pt, laura.l.cavalcante@inesctec.pt, \\ vmiranda@inesctec.pt
}

\begin{abstract}
In order to reduce the curtailment of renewable generation in periods of low load, operators can limit the import net transfer capacity (NTC) of interconnections. This paper presents a probabilistic approach to support the operator in setting the maximum import NTC value in a way that the risk of curtailment remains below a pre-specified threshold. Main inputs are the probabilistic forecasts of wind power and solar PV generation, and special care is taken regarding the tails of the global margin distribution (all generation - all loads and pumping), since the accepted thresholds are generally very low. Two techniques are used for this purpose: interpolation with exponential functions and nonparametric estimation of extreme conditional quantiles using extreme value theory. The methodology is applied to five representative days, where situations ranging from high maximum $\mathrm{NTC}$ values to $\mathrm{NTC}=\mathbf{0}$ are addressed. Comparison of the two techniques for modeling tails is also comprised.
\end{abstract}

Keywords-Renewable energy, forecasting, risk, net transfer capacity, probabilistic, transmission network, TSO

\section{INTRODUCTION}

The integration of large amounts of renewable energy sources, the completion of the internal electricity market, as well as a new energy generation mix, which means more fluctuating renewable energy in the system and less conventional fossil fuel generation, requires the development of new reliability methodologies to compute reserve capacity on a transmission network. Traditionally, power system reliability studies for static and operating reserve capacity were mainly concerned with the risk of failing to satisfy the load, as the result of loss of generation due to forced outages and load forecast errors [1].

When the integration level of renewable energy sources (RES) in the power system becomes noteworthy, the impact of RES uncertainty on the load-generation balance becomes critical. For long-term reserve capacity planning (or monitoring of the adequacy of supply) several methodologies were proposed in the literature to include RES uncertainty, see [2] and [3]. For short-term operating reserve setting, the

\author{
N. Machado, P. Marques, F. Matos \\ REN-Redes Energéticas Nacionais SA \\ Lisboa, Portugal \\ nelio.machado@ren.pt,paulo.marques@ren.pt, \\ fernando.matos@ren.pt
}

current state of the art consists in including information from RES uncertainty forecasts (e.g., forecasted quantiles, temporal trajectories) in risk-based decision tools [4]. Recent work has been focused in quantifying intra-hourly RES variability by including probabilistic information about load and RES variability [5].

All these studies and methodologies are mostly focused in upward reserve capacity and little attention was given to scenarios with extreme RES generation and high probability of having more generation than load in the system. In noninterconnected systems and in the absence of sufficient flexibility from the demand-side (e.g., demand response products, storage), these scenarios result in curtailment of RES generation, which is not desired due to environmental (and regulatory in some case) reasons. In interconnected systems, restricting in advance the maximum import value of the Net Transfer Capacity (NTC) with neighboring control areas might mitigate this risk. However, due to the uncertainty of RES generation and considering that restricting import NTC value could also lead to market restrictions on boundary systems, a risk-based methodology is required.

The current literature on this topic is mainly driven to study the adequacy of the current European transmission network and evaluate the necessity for future expansions from a planning domain perspective. For instance, Farahmand et al. in [6] conducted a cost-benefit analyses to assess the transmission grid needed to integrate wind generation distant from load centers and explore hydropower flexibility in Nordic countries; Hagspiel et al. in [7] applied copula theory to model the impact of wind generation in the European interconnected network in terms of risk of overloading and to prioritize necessary grid reinforcements.

In the operating time domain, several authors studied the impact of wind power generation (and its forecasts) in the NTC. For instance, Salic and Rebours in [8] conducted a statistical analysis with regression models to evaluate the impact of the day-ahead forecasts of German wind generation on the day-ahead NTC from Germany to France, which results 
show a significant impact on the NTC; Rious et al. in [9] studied the impact of German wind power generation on the Available Transfer Capacity (ATC) calculation between France and Belgium and concluded that the impact varies with the geographical distribution of the wind generation in Germany.

Perninge in $[10]$ developed a Monte Carlo simulation method to estimate the risk of violating the Total Transfer Capacity (TTC), by including probability distributions from load, generation and grid state. The main limitation of this work is that probabilistic information from forecasting tools is not included in the model. Furthermore, scenarios with extreme RES generation are not covered.

The present paper addresses these limitations by adapting a probabilistic methodology described in [11] for setting operating reserve requirements from load and RES probabilistic forecasts in order to estimate the maximum import value in the cross-border interconnection. The original contribution from this paper is a risk-based methodology that determines the maximum value for the import NTC that respects a maximum limit (risk threshold defined by the decision-maker) the probability of wasting renewable generation. Results from a real-world operational test for the Portuguese power system are presented, particularly for specific days with high wind generation levels.

The remaining of the paper is organized as follows: section II describes the risk-based methodology to estimate the maximum import NTC; section III presents the results for Portugal for days with extreme RES generation levels; finally, section IV presents the conclusions.

\section{RISK-BASED METHODOLOGY}

\section{A. Components of the Methodology}

Fig. 1 depicts the input data, components and output of the proposed methodology. Every day, the Transmission System Operator (TSO), at day D and before daily market for day D+1, can revise (if necessary) the maximum hourly import NTC value for the next day $\mathrm{D}+1$. The aim of the proposed methodology is to support the TSO to determine the maximum import NTC value that ensures that a certain risk level is not exceeded. Risk here means the probability of having more generation than load in the system, as a result of uncertainty in RES and load forecasting.

It is important stress that the objective is not to discuss the complementary problem that is how much operating reserve is required to limit the probability of loss of load and RES curtailment, which is covered by [11].

The input data consists of the following variables: wind power, small-hydro, combined heat and power (CHP) and solar power probabilistic forecasts, total load in the system, minimum conventional generation capacity that must be in the system due to stability reasons, and maximum pump storage power capacity of the system (considering operational constraints such as water reservoir levels), for each look-ahead time step of day $\mathrm{D}+1$.
The selection of these inputs is intended to create an extreme operating scenario with conventional generation at the minimum level and pump storage at its maximum value in order to study the probability of having surplus of RES generation.

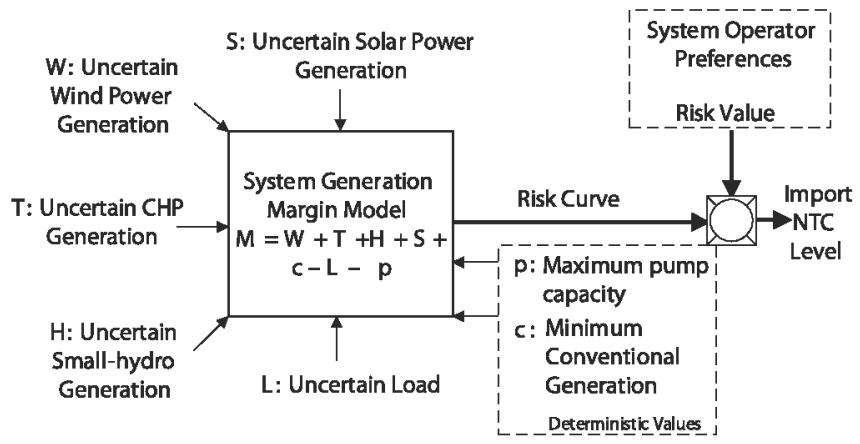

Fig. 1. Structure of the proposed method for setting the maximum import NTC.

The core of the methodology is based on the calculation of the probability distribution of the balance system equation, given by the difference between generation and load (including maximum pump capacity).

This tool uses a probabilistic approach to build the system generation's margin distribution and its probability distribution results from convolving the probability mass functions of each random variable component, as in [11]. An important assumption is independence between each random variable (i.e., forecast errors). For the real case-study described in section III, the Spearman's rank correlation between wind power and load was -0.025 forecast errors, and between wind and solar power errors was -0.077 . Therefore, this assumption is perfectly reasonable for this case. Otherwise, copula theory can be used for modelling the dependency structure of forecast errors [7].

The output is a suggestion of the maximum import NTC value for each hour of the next day that ensures a certain maximum risk fixed by the operator. Furthermore, information about the forecast interval composed by the quantiles $5 \%$ and $95 \%$ (centered in the median) is also presented to the operator in order communicate the level of uncertainty (of sharpness) of the probabilistic forecasts. With this approach, and in hours where the suggested maximum import NTC is low, the operator has additional information about the level of uncertainty associated to each generation technology.

\section{B. Input Data Modelling}

a) Uncertainty modelling: Probabilistic forecasts, represented by a set of quantiles ranging between $5 \%$ and $95 \%$, are generated with a statistical model (see [12] and [13] for more details) to wind power, small-hydro, CHP and solar power since these generation technologies are under a feed-in support scheme in Portugal (called special regime generation) and are the last in the merit-order of curtailment actions. This representation follows the methodology proposed in [11].

A probability mass function ( $\mathrm{pmf}$ ) is derived from the forecasted cumulative distribution function since it enables the application of convolution techniques to the random variables 
(see section II.C). The following procedure is adopted to create pmf: (a) the forecasted cumulative function is linearly interpolated; (b) from the interpolated function, the value of equally spaced quantiles is derived and the corresponding probability is assigned to the central point between the two quantiles' values.

The load forecasting uncertainty is modeled by a Gaussian distribution centered in the point forecast and standard deviation calculated from the Mean Absolute Percentage Error (MAPE) [11].

For the pump storage power capacity and conventional generation only deterministic information is considered. However, the proposed methodology is compatible with the inclusion of unplanned outages in a capacity outage probability table

b) Forecasting the distribution tails: modelling of the distribution tails is a critical factor to a proper modelling of the risk. The regulatory framework imposes risk aversion to TSO, thus the admissible risk level is always below 1\% which estimation quality highly depends from the distribution tails' estimation.

Two different models were explored to model the tails: (a) interpolation with exponential functions as used in [11] and [14], but estimating the rate parameter as in [15] and then using it to obtain the thickness parameter; (b) nonparametric estimation of extreme conditional quantiles using extreme value theory [16].

Very briefly, the first method considers that the range of generation variables is within 0 and 1 (the data is therefore normalized by dividing by the maximum power installed) and so the physical constrains of generation are respected. First, a $\delta$ value is chosen and all points are assumed to be in the interval $[\delta, 1-\delta]$, otherwise they are replaced by values spaced by $\delta$, beginning in $\delta$ if less than $\delta$ or ending in $1-\delta$ if greater than $1-\delta$. The values are then sorted and are supposed to be different, otherwise they are subsequently increased by $\delta$; in this work, $\delta$ is chosen to be 0.01 .

After that, using the 90 -quantiles estimated in the first phase (ranging between $5 \%$ and $95 \%$ with $1 \%$ increments), the estimation of the cumulative distribution function is extended to the estimated quantiles of Eq. 1.

$$
\begin{cases}0, & y=0 \\ \rho \cdot \exp \left(\frac{q^{(5 \%)}}{y} \cdot \ln \left(\frac{0.05}{\rho}\right)\right), & y=i . \delta, i \in\left\{1, \ldots,\left\lfloor\frac{q^{(5 \%)}}{\delta}\right]\right\} \\ \alpha_{N}, & y=q^{\left(\alpha_{N} \%\right)}, \alpha_{N} \in\{5, \ldots, 95\} \\ 1-\rho \cdot \exp \left(\frac{1-q^{(95 \%)}}{1-y} \cdot \ln \left(\frac{1-0.95}{\rho}\right)\right), & y=q^{(95 \%)}+j . \delta, \\ & j \in\left\{1, \ldots,\left\lfloor\frac{1-q^{(95 \%)}}{\delta}\right]\right\} \\ 1, & y=1\end{cases}
$$

An important parameter in this method is the $\rho$ parameter that corresponds to the thickness parameter for the exponential extrapolation of generation variable. However, computing more than one value is needed of this parameter because it is not correct to assume that an observation, where the $50 \%$ quantile estimation is small, has the same mean/ thickness generation than an observation where the estimated $50 \%$ quantile takes a higher value. To try mitigating this limitation, the historical sample is divided into 10 intervals according to the sampling quantiles of the estimated quantile $50 \%$, spaced by $10 \%$ increments. With these intervals, the $\rho_{[\mathrm{a}, \mathrm{b}]}$ parameter is estimated by

$$
\rho_{[a, b]}=\frac{\sum_{i=1}^{N} I_{[a, b]}\left(q_{i}^{(50 \%)}\right) y_{i}}{N}
$$

where $a, b$ are two successive sampling quantiles computed, $\boldsymbol{I}$ is a indicatrix function

$$
I_{[a, b]}\left(q_{i}^{(50 \%)}\right)=\left\{\begin{array}{l}
0, \text { if } q_{i}^{(50 \%)} \notin[a, b] \\
1, \text { if } q_{i}^{(50 \%)} \in[a, b]
\end{array}\right.
$$

and the $q_{i}^{(50 \%)}$ is the estimated quantile $50 \%$ from the historical observation which the observed generation were $y_{i}$, $i \in\{1, \ldots, N\}$. Note that for the first interval the lower limit is 0 , and for the last interval the upper limit is the maximum installed power.

The second method is also a conditional estimation assuming that the power generation variable $\mathrm{Y}$ is conditioned by a second variable $X$. In our case study, the variable $X$ is considered the quantile $50 \%$ of the first phase of the quantile estimation.

The first step is to compute a quantile regression that reduces the sample size and uses the historical observations more similar with the present forecast [16]. With the resulting sample, the tail index parameter is estimated using the general maximum likelihood method and the correspondent extreme conditional quantiles formula [16].

The methods were evaluated and compared using the quantile score and calibration measures from [17]. The corresponding results are presented in Appendix. The conclusion is that the first method better models our data in the tails, and it will be used for the results presented in section IV.

\section{Downward System Margin Estimation}

Firstly, the tool computes the probability distribution of the system's total generation for each day-ahead time step, by summing the wind $(W)$, thermal $(T)$, hydro $(H)$ and solar $(S)$ generation forecast uncertainty, and the deterministic value of minimum conventional generation (c). Secondly, the probability distribution of the system balance equation is obtained by subtracting the load uncertainty $(L)$ and the deterministic value of maximum pump capacity $(p)$.

A convolution with the Fast Fourier Transform (FTT) is performed in pairs. We first compute the probability mass function of the sum between wind and thermal generation $\left(G_{1}=W+T\right)$ as showed in (1). Assuming independence, this sum can be computed convolving the two random variables: 


$$
P_{G_{1}}(W+T=z)=\sum_{k=-\infty}^{\infty} P_{W}(W=z-k) \cdot P_{T}(T=k)
$$

The next step is to sum the hydro generation $\left(G_{2}=W+T+H=G_{1}+H\right)$ :

$$
P_{G_{2}}\left(G_{1}+H=z\right)=\sum_{k=-\infty}^{\infty} P_{G_{1}}\left(G_{1}=z-k\right) \cdot P_{H}(H=k)
$$

Analogously, we sum the solar generation and obtain the mass function of the random variable $G_{3}=W+T+H+S$. Then, the total generation $(G)$ of the system is computed integrating the $c$ constant $(G=W+T+H+S+c)$.

After which the mass function of the difference between total generation and load is computed as:

$$
P_{G}(G-L=z)=\sum_{k=-\infty}^{\infty} P_{G}(G=z+k) \cdot P_{L}(L=k)
$$

Finally, the system margin is obtained subtracting the constant $p$. An example of system margin generation equation probability mass distribution is illustrated in Fig. 2.

The classical measures of reliability can be calculated from the system generation margin distribution, e.g., probability of wasting special regime generation (PWG). In our work, the PWG measure was used for pragmatic reasons: the risk value can be interpreted as the probability that the RES (wind, thermal, hydro and solar generation) must be curtailed or the energy exported increased.

In the example of Fig. 2, if the TSO takes an import NTC level equal to 0 , the probability of having excess of generation is $0 \%$. On the other hand, if the TSO decides to set the import NTC to 1000 MW then the curve presented in Fig. 3 is translated $1000 \mathrm{MW}$ to the right, as can be seen in Fig. 3, and the associated risk becomes $0.5 \%$ (red region in the figure). In this case, if the TSO is comfortable with a PWG equal to $0.5 \%$, the import NTC capacity can be set to $1000 \mathrm{MW}$.

In order to compute the suggested level of the NTC with that metric, it is possible to directly use the cumulative distribution of $M$ and calculate the greatest $I$ value such that $P(M \leq I) \leq 1-\alpha$, where $\alpha$ is the value of the risk measure fixed by the TSO. Two possible scenarios can occur to the signal of $I$ :

- Negative $I$ : the probability of having more generation than load is less than the risk value so the NTC tool should suggest a $-I$ value for the import NTC level.

- Positive $I$ : the probability of generation exceeding the load in the system is already greater than the risk value, therefore the NTC tool should suggest a zero value for NTC level. In this case, information about the expected energy curtailment can be calculated by summing the product of the positive values of $I$ by its probability. This gives to the operator additional information about the expected magnitude of the special regime generation curtailment ${ }^{1}$.

\section{Decision Rules}

The adopted methodology requires the choice of a threshold value for the maximum acceptable risk. The TSO might find that the suggested maximum import NTC level leads to excessive risk, in which case the TSO might want to consider reducing the suggested level. Therefore, the TSO must continuously analyze the results to better define the relation between risk and value for the maximum import NTC.

With this approach, a risk/import NTC curve is constructed and the import level automatically computed when a new risk level is fixed. Fig. 4 and Fig. 5 shows the correspondent curve risk/ maximum import NTC level for the situation represented by Fig. 2. In this case, if the SO fixes a risk value of $0.5 \%$ (corresponding to a cumulative probability of $99.5 \%$ in margin system distribution), the suggested import NTC level is $1150 \mathrm{MW}$. For a risk level of $1 \%$, the suggestion is approximately $1300 \mathrm{MW}$. These two examples correspond to the first scenario of negative $I$ in the cumulative distribution function.

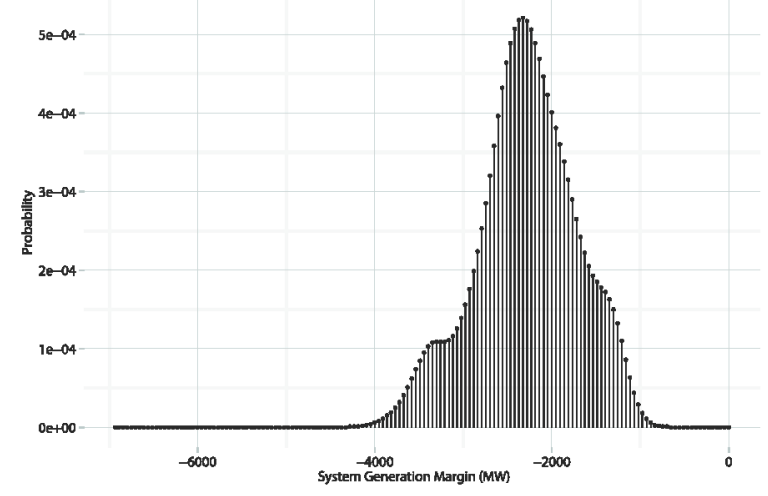

Fig. 2 Probability mass function of the margin for a specific time step.

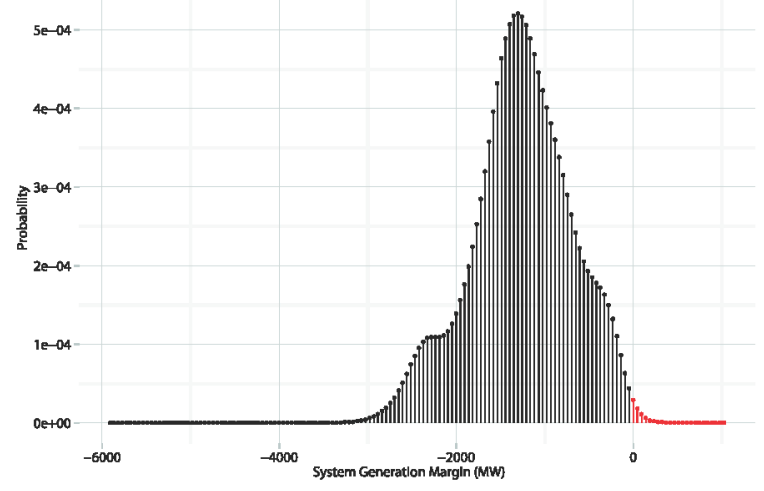

Fig. 3 Probability mass function of the margin for a specific time step and for a maximum import NTC of $1000 \mathrm{MW}$.

\footnotetext{
${ }^{1}$ Risk index analogous to the Expected Energy Not Supplied (EENS).
} 


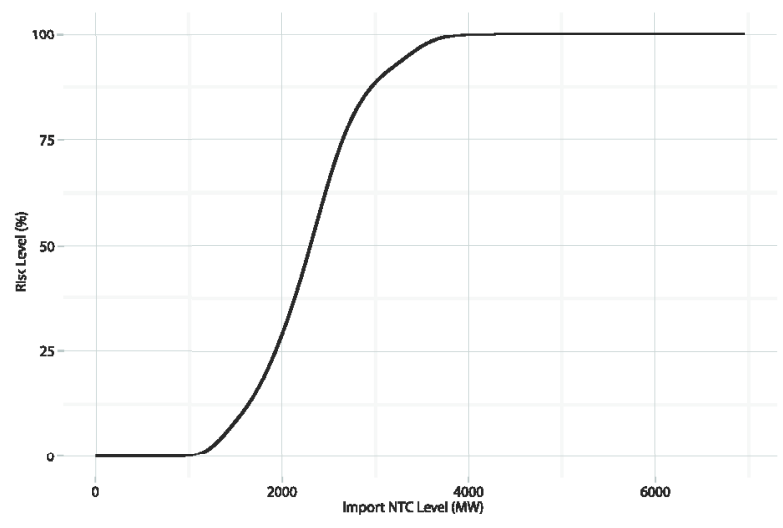

Fig. 4 Curve risk/ maximum import NTC level for a specific time step.

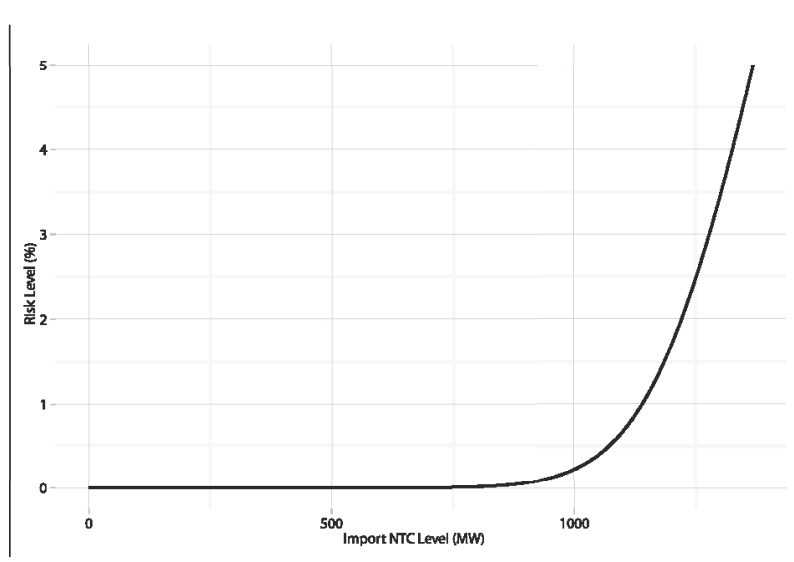

Fig. 5 Negative tail zoom of Fig. 4.

\section{CASE-STUDY}

\section{A. Portuguese Power System}

The capacity mix in the Portuguese power system in the end of 2015 was: 5,724 MW of large hydropower plants, $5,585 \mathrm{MW}$ of thermal generation and 7,224 MW of generation remunerated by feed-in tariffs support schemes. The generation under a tariff remuneration scheme consists of small-hydro (422 MW), CHP (1548 MW), wind power (4826 MW), and photovoltaic (429 MW) - totalizing $7224 \mathrm{MW}$. The installed pump storage power is $1618 \mathrm{MW}$.

In 2015 , the maximum peak power was $8618 \mathrm{MW}$. The load-factor of the wind power generation was $32 \%$ and $10 \%$ for solar power in $2015 ; 23 \%$ of the annual consumption (48.964 TWh in 2015) was supplied by wind power, in contrast to $18 \%$ from large hydropower plants and $39 \%$ from thermal generation.

Fig. 6 depicts the percentage of total load supplied by wind generation in five days between December 2015 and March 2016.

For the same days, Fig. 7 depicts the percentage of total load supplied by special regime generation.

These days constitute good examples of situations where special regime generation, and in some cases wind power alone, are sufficient to meet the load, or even exceed it. In these extreme circumstances, only curtailment (or export) is possible, but when load still exceeds generation, a certain amount of power can be imported, so it is possible to set a maximum import $\mathrm{NTC}>0$, maintaining the risk of curtailment of special regime generation below the threshold set by the operator.

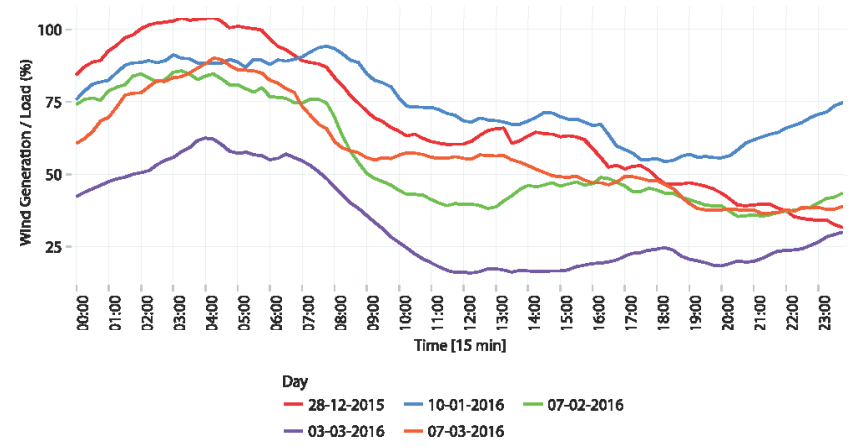

Fig. 6. Percentage of total load supplied by wind generation in five days between December 2015 and March 2016.

During these days, pump storage power also played an important role in RES integration. For instance, in 28-12-2015 a maximum of $1264.9 \mathrm{MW}$ (78\% of installed capacity) was reached at $02: 15$, during which only $11 \mathrm{MW}$ were being exported to Spain.

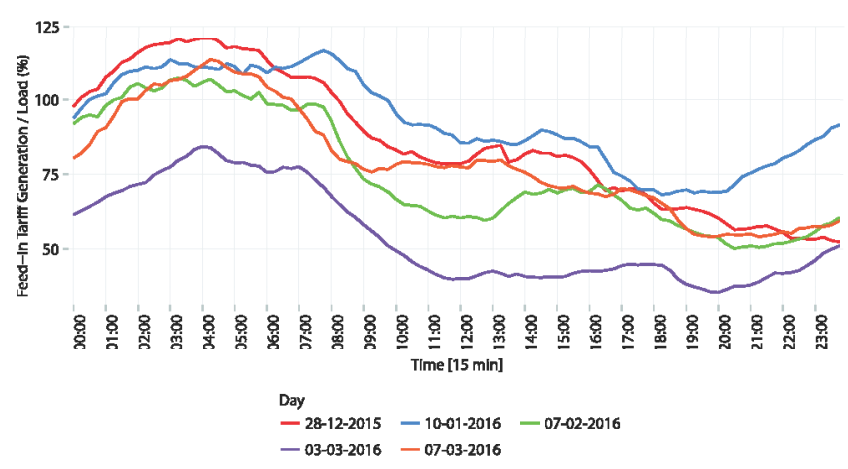

Fig. 7. Percentage of total load supplied by special regime generation in five days between December 2015 and March 2016.

\section{B. Numerical Results}

The days mentioned in Section III.A were chosen to illustrate the methodology. In some hours of those days, the total generation was very high and, as expected, the import NTC tool suggests a zero import NTC level.

In Fig. 8 the maximum import NTC tool suggestions are presented relatively to PWG levels between $0.01 \%$ (light green) and 5\% (red). Note that a negative value leads to a recommendation of NTC equal to 0 . Furthermore, the curve that corresponds to the balance equation value (see Fig. 1) using the observed values of special regime generation and load is also presented. Note that this type of fan chart is particularly useful to communicate the risk associated to a set of alternative decisions to dispatch center' operators.

In all these days, the highest proportion values between special regime generation and load were registered in the time 
horizon between 1:00AM and 6:00AM. And, with the exception of day 03-03-2016, in all of these hours, these proportions were greater than $100 \%$.
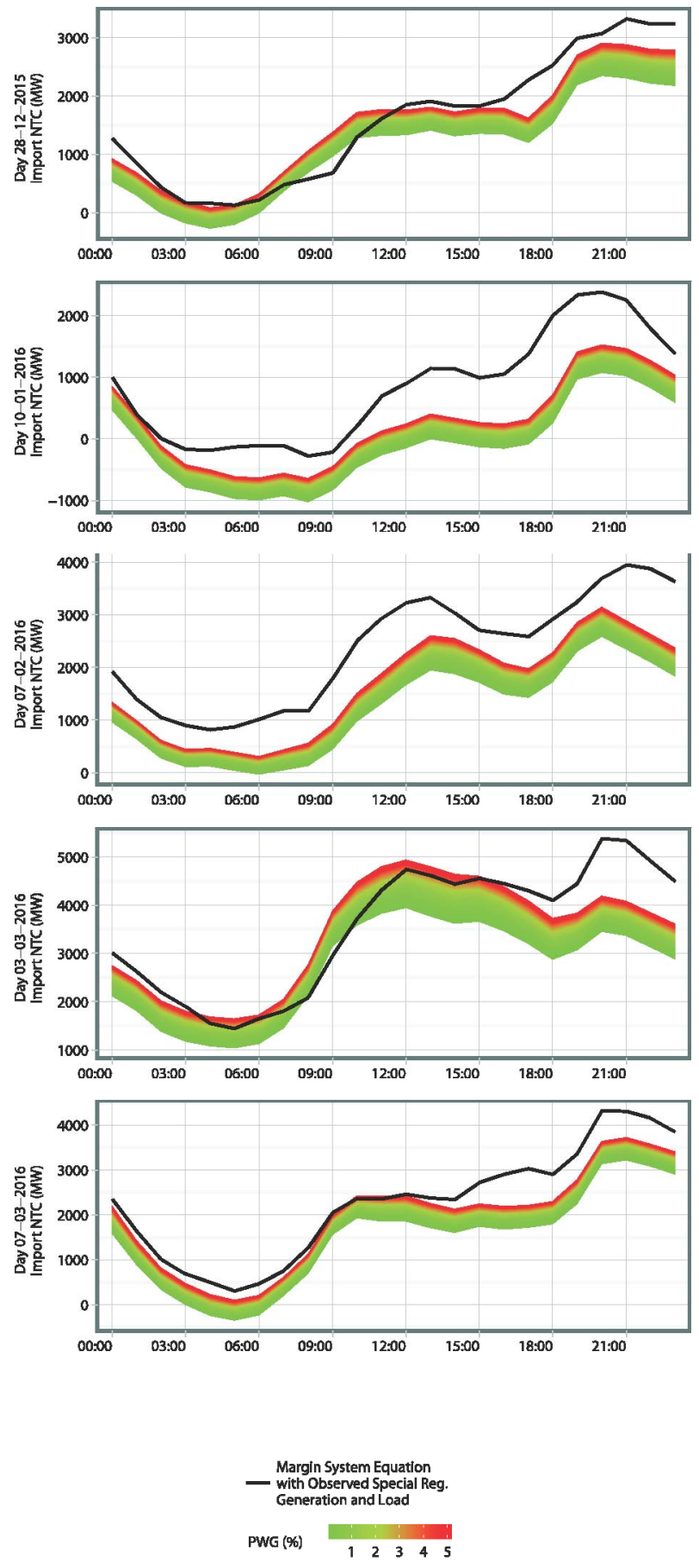

Fig. 8. Import NTC Levels suggested to a PWG between $0.01 \%$ and $5 \%$.

At day 03-03-2016, the special regime generation was less than the load so it is not a surprise that the suggested a maximum import NTC level would be greater than in the rest of the days. In fact, the maximum import NTC value that ensures that a PWG of 5\% is not surpassed is greater relatively to the other days. As can be observed, an increase in the proportion between special regime and load is accompanied by a decrease of the import NTC level. Analogously, in the hours in which a decreasing of the proportion occurred, the import NTC tool estimated mostly an increase of the required import NTC.

Some important remarks about the results: at days 28-122015 and 03-03-2016, the solid curve has registered a big deviation relatively to the represented quantiles. This means that the expected value of the computed pmf of load uncertainty and/ or RES generation has a considerable deviation relative to the observed values. During the night period of day 10-01-2016, the import NTC capacity was negative (which means that the limit should be set to zero) for any risk value between $5 \%$ and $0.01 \%$. In this case, even with the import NTC set to zero there is a high risk of generation curtailment. The methodology can also inform the operator about the expected energy curtailment (or generation that needs to be exported), e.g. $282 \mathrm{MWh}$ at 6:00, $300 \mathrm{MWh}$ at 7:00.

In the spccific casc of 28-12-2015, this difference was essentially due to load uncertainty. As detailed above, the load forecasting uncertainty is modeled by a Gaussian distribution centered in the point forecast and, in that day, the point forecasts has a big deviation relatively to the observed values. At 10:00 AM, for example, a difference was registered of almost $700 \mathrm{MW}$ between these two measures; this means that the NTC tool considered more load than the observed and so allocated more import NTC level than adequate.

In the other end, at 03-03-2016, the largest deviation was registered in the RES generation. In 9:00AM, the biggest deviation registered had a difference of 1000MW between the expected RES energy and the observed value.

\section{CONCLUSIONS}

This paper presents a decision support method to help the system operator in defining a Indaximum import NTC value by taking into account an extreme scenario where the required pump storage power level is assumed to be the technical available and that the conventional generation will take the minimum possible value (i.e., must-run units). The methodology also considers the wind, thermal, hydro and solar power forecast uncertainty and load forecast uncertainty. With these components, the system margin pmf is modeled and a maximum value of import NTC that ensures that a certain risk of curtailment is not surpassed can be obtained.

The results obtained for five days, between Dec. 2015 and Mar. 2016, with extreme integration levels of special regime generation (mainly wind power) showed the following conclusions: (a) under extreme RES integration, the event associated to generation surplus becomes also critical, even in the present high storage capacity; (b) uncertainty forecasts can be useful to support decisions at the dispatch center level, but additional work is required to better communicate information about uncertainty and related decisions; (c) the modelling of probability distribution tails is critical for decision-makers, such as TSO, that are only willing to take a very low risk.

Future work consists in improving the tails forecasting with conditional extreme value theory and develop innovative 
approaches to communicate information about uncertainty to dispatch center operators.

\section{APPENDIX - TAILS' ESTIMATION QUALITY}

The following tables show the evaluation results of the two methods considered to estimate the extreme quantiles in the tails. The model parameters were estimated using 120 days of hourly observations and then tested using 21 days, for a total of 504 observations. The exponential interpolation is denoted by EI and conditional extreme value theory values by CEVT.

TABLE I

AVERAGE VALUES OF THE QUANTILE SCORE.

\begin{tabular}{c|cc}
\hline & $\begin{array}{c}\text { Lower } \\
\text { Tail }\end{array}$ & $\begin{array}{c}\text { Upper } \\
\text { Tail }\end{array}$ \\
\hline CEVT & -126 & -153 \\
EI & -95 & -123 \\
\hline \multicolumn{3}{c}{ TABLE II }
\end{tabular}

LOWER TALL CALIBRATION.

\begin{tabular}{c|cccccc}
\hline Quantile & $2 \%$ & $1 \%$ & $0.5 \%$ & $0.1 \%$ & $0.05 \%$ & $0.01 \%$ \\
\hline CEVT & 0.0200 & 0.0100 & 0.0050 & 0.0010 & 0.0005 & 0.0001 \\
EI & 0.0081 & 0.0080 & 0.0050 & 0.0010 & 0.0005 & 0.0001 \\
\hline
\end{tabular}

TABLE III

UPPER TAIL CALIBRATION.

\begin{tabular}{c|cccccc}
\hline Quantile & $98 \%$ & $99 \%$ & $99.5 \%$ & $99.9 \%$ & $99.95 \%$ & $99.99 \%$ \\
\hline CEVT & -0.0200 & -0.0100 & -0.0050 & -0.0010 & -0.0005 & -0.0001 \\
EI & -0.0002 & -0.0021 & -0.0030 & -0.0010 & -0.0005 & -0.0001 \\
\hline
\end{tabular}

Table I shows the results obtained with the quantile score presented in [17]. From the Table I it can be concluded that the quality of the results in terms of mean quantile scoring was better in the EI method. However, the calibration, presented in Tables II and II, is also an important measure and the conjoint analysis of the two measures is needed to evaluate the quality of the methods. So, in terms of calibration, the exponential interpolation is also the method with better performance.

Note that, for the nominal proportions $0.01 \%, 0.05 \%$, $0.1 \%, 0.5 \%$ and $99.5 \%, 99.9 \%, 99.95 \%, 99.99 \%$, the calibration have the same value in the two methods but the quantile scoring of the exponential interpolation is the smallest possible value. This means that the sharpness of the estimated quantiles computed by the CEVT method is higher than those estimated by the EI method.

\section{ACKNOWLEDGMENT}

This work was funded by the ERDF - European Regional Development Fund through the Operational Programme for Competitiveness and Internationalisation - COMPETE 2020 Programme within project «POCI-01-0145-FEDER-006961», and by National Funds through the FCT - Fundação para a Ciência e a Tecnologia (Portuguese Foundation for Science and Technology) as part of project UID/EEA/50014/2013. The authors also acknowledge the support of REN (Redes Energéticas Nacionais SA) for developing the methodology.

\section{REFERENCES}

[1] R. Billinton and R.N. Allan, Reliability Evaluation of Power Systems, New York and London: Plenum Press, 1984.

[2] M. Matos, J.A. Peças Lopes, M. Rosa, R. Ferreira, A. Leite da Silva, W. Sales, et al., "Probabilistic evaluation of reserve requirements of generating systems with renewable power sources: The Portuguese and Spanish cases," International Journal of Electrical Power \& Energy Systems, vol. 31(9), pp. 562-569, 2009.

[3] R. Billinton and G. Bai, "Generating capacity adequacy associated with wind energy," IEEE Transactions on Energy Conversion, vol. 19(3), pp. 641-646, 2004.

[4] H. Holttinen, M. Milligan, E. Ela, N. Menemenlis, J. Dobschinski, B. Rawn, R.J. Bessa, D. Flynn, E.G. Lazaro, N. Detlefsen, "Methodologies to determine operating reserves due to increased wind power," IEEE Transactions on Sustainable Energy, vol. 3(4), pp. 713-723, Oct. 2012.

[5] H. Nosair, F. Bouffard, "Flexibility envelopes for power system operational planning," IEEE Transactions on Sustainable Energy, vol. 6(3), pp. 800-809, 2015.

[6] H. Farahmand, S. Jaehnert, T. Aigner, D. Huertas-Hernando, "Nordic hydropower flexibility and transmission expansion to support integration of North European wind power," Wind Energy, vol. 18(6), pp, 1075-1103, 2015.

[7] S. Hagspiel, A. Papaemannouil, M. Schmid, G. Andersson, "Copulabased modeling of stochastic wind power in Europe and implications for the Swiss power grid," Applied Energy, vol. 96, pp. 33-44, 2012.

[8] G. Salic, Y. Rebours, "Impact of German wind generation forecasts on net transfer capacities," in Proc. of the 2011 8th International Conference on the European Energy Market (EEM), pp. 635-640, Zagreb, Croatia, 2011.

[9] V. Rious, J. Usaola, M. Saguan, J. Glachant, P. Dessante, "Assessing available transfer capacity on a realistic European network: impact of assumptions on wind power generation," in Proc. of the lst International Conference on of the Infrastructure Systems and Services: Building Networks for a Brighter Future (INFRA), Rotterdam, Holland, 2008.

[10] M. Perninge, "Modeling the uncertainties involved in net transmission capacity calculation," KTH School of Electrical Engineering, Licentiate Thesis, 2009.

[11] M.A. Matos, R.J. Bessa, "Setting the operating reserve using probabilistic wind power forecasts," IEEE Transactions on Power Systems, vol. 26(2), pp.594-603, 2011.

[12] R.J. Bessa, V. Miranda, A. Botterud, J. Wang, and Emil M. Constantinescu, "Time adaptive conditional kernel density estimation for wind power forecasting," IEEE Transactions on Sustainable Energy, vol. 3(4), pp. 660-669, Oct. 2012.

[13] H. Nielsen, H. Madsen, T.S. Nielsen, "Using quantile regression to extend an existing wind power forecasting system with probabilistic forecasts," Wind Energy, vol. 9(1-2), pp. 95-108, Jan-Apr. 2006.

[14] P. Pinson, G. Papaefthymiou, B. Klockl, H. A. Nielsen, and H. Madsen, "From probabilistic forecasts to statistical scenarios of short-term wind power production," Wind Energy, vol. 12, no. 1, pp. $51-62,2009$.

[15] T. Jónsson, P. Pinson, H. Madsen, H. Nielsen, "Predictive densities for day-ahead electricity prices using time-adaptive quantile regression," Energies, vol. 7, pp. 5523-5547, 2014.

[16] J. Beirlant, T. Wet, Y.Goegebeur, "Nonparametric estimation of extreme conditional quantiles," Journal of Statistical Computation and Simulation, vol.74, pp. 567-580 2004.

[17] P. Pinson, H. A. Nielsen, J. K. Møller and H. Madsen, "Nonparametric probabilistic forecasts of wind power: Required properties and evaluation," Wind Energy, vol. 10, pp.497-516, 2007. 